\title{
Constants of motion associated with canonoid transformations for non-autonomous systems
}

\author{
G.F. Torres del Castillo \\ Instituto de Ciencias Benemérita Universidad Autónoma de Puebla, \\ 72570 Puebla, Pue., México \\ R. Azuaje \\ Facultad de Ciencias Físico Matemáticas, Benemérita Universidad Autónoma de Puebla, \\ 72570 Puebla, Pue., México
}

Received 17 August 2021; accepted 4 October 2021

\begin{abstract}
We consider non-autonomous systems of ordinary differential equations that can be expressed in Hamiltonian form in terms of two different coordinate systems, not related by a canonical transformation. We show that the relationship between these coordinate systems leads to a, possibly time-dependent, tensor field, $S_{\beta}^{\alpha}$, whose eigenvalues are constants of motion. We prove that if the Nijenhuis torsion tensor of $S_{\beta}^{\alpha}$ is equal to zero then the eigenvalues of $S_{\beta}^{\alpha}$ are in involution, and that these eigenvalues may be in involution even if the Nijenhuis tensor is not zero.
\end{abstract}

Keywords: Integrable systems; non-autonomous systems; canonoid transformations; Nijenhuis torsion tensor.

DOI: https://doi.org/10.31349/RevMexFis.68.020706

\section{Introduction}

The integrability of the systems of ordinary differential equations found in classical mechanics has been studied for a long time. For a system of ordinary differential equations written in the form of the Hamilton equations with $n$ degrees of freedom, the knowledge of $n$ functionally independent constants of motion in involution allows us to reduce to quadratures the solution of the entire set of equations (see, e.g., Refs. [1-3]).

The so-called bi-Hamiltonian systems have been studied in connection with the integrability mentioned above. It has been shown that if an autonomous system of equations can be written in the form of the Hamilton equations making use of two different symplectic structures, satisfying certain compatibility condition, then one can find a set of constants of motion in involution, which are related by means of recurrence operators (see, e.g., Refs. [4-8]).

On the other hand, in Refs. [9-11] it has been shown that it is possible to find constants of motion by considering the so-called canonoid transformations admitted by a Hamiltonian corresponding to the system of interest. Here we reserve the name canonoid transformation for a non-canonical coordinate transformation that preserves the form of the Hamilton equations for a given Hamiltonian.

The aim of this paper is to extend the results about bi-Hamiltonian systems already mentioned to the nonautonomous case, starting from the canonoid transformations. We show that for a given Hamiltonian system with Hamiltonian function possibly depending explicitly on time, and with Hamilton equations expressed in terms of a set of canonical coordinates, or of a Poisson bracket, each canonoid transformation leads to a second Poisson bracket related to the first one by means of a tensor field $S_{\beta}^{\alpha}$, such that the trace of the each power of the matrix $\left(S_{\beta}^{\alpha}\right)$ is a constant of motion (which can depend explicitly on the time). We also show that if the Nijenhuis torsion tensor of $S_{\beta}^{\alpha}$ is equal to zero then the traces of the powers of $\left(S_{\beta}^{\alpha}\right)$ (or the eigenvalues of $\left(S_{\beta}^{\alpha}\right)$ ) are in involution, but they may not be functionally independent. We give examples of systems for which the Nijenhuis torsion tensor of $S_{\beta}^{\alpha}$ is different from zero, but the traces of the powers of $\left(S_{\beta}^{\alpha}\right)$ are constants of motion in involution.

In Sec. 2 we present some basic facts about canonoid transformations. In Sec. 3 we review the definition of the (autonomous) bi-Hamiltonian systems and we show the local equivalence between the bi-Hamiltonian systems and the canonoid transformations in the autonomous case. We then show that the integrability properties of the autonomous biHamiltonian systems can be extended to the non-autonomous systems that admit a canonoid transformation. In Sec. 4 we present some explicit examples of canonoid transformations for non-autonomous systems.

\section{Canonoid transformations}

Let $\left(q^{1}, \ldots, q^{n}, p_{1}, \ldots, p_{n}\right)$ be canonical coordinates for a Hamiltonian system with Hamiltonian function $H\left(q^{i}, p_{i}, t\right)$, which may depend explicitly on time. We know that the dynamics of the system is defined by a vector field $\mathbf{X}$ such that $\mathbf{X}\lrcorner\left(\mathrm{d} p_{i} \wedge \mathrm{d} q^{i}-\mathrm{d} H \wedge \mathrm{d} t\right)=0$, where $\lrcorner$ denotes contraction; this vector field has the form

$$
\mathbf{X}=\frac{\partial H}{\partial p_{i}} \frac{\partial}{\partial q^{i}}-\frac{\partial H}{\partial q^{i}} \frac{\partial}{\partial p_{i}}+\frac{\partial}{\partial t}
$$

with summation over repeated indices. The integral curves of $\mathbf{X}$ are the solutions of the Hamilton equations (see, e.g., Ref. [12]). 
We are interested in canonoid transformations that may depend explicitly on time, that is, coordinate transformations of the form $Q^{i}=Q^{i}\left(q^{j}, p_{j}, t\right), P_{i}=P_{i}\left(q^{j}, p_{j}, t\right)$ that preserve the form of the Hamilton equations (see, e.g., Ref. [13]), which means that $\mathbf{X}\lrcorner\left(\mathrm{d} P_{i} \wedge \mathrm{d} Q^{i}-\mathrm{d} K \wedge \mathrm{d} t\right)=0$, for some function $K$.

A straightforward computation gives

$$
\begin{aligned}
\mathrm{d} P_{i} \wedge \mathrm{d} Q^{i} & -\mathrm{d} K \wedge \mathrm{d} t \\
& =\left(\frac{\partial P_{i}}{\partial p_{j}} \mathrm{~d} p_{j}+\frac{\partial P_{i}}{\partial q^{j}} \mathrm{~d} q^{j}+\frac{\partial P_{i}}{\partial t} \mathrm{~d} t\right) \wedge\left(\frac{\partial Q^{i}}{\partial p_{s}} \mathrm{~d} p_{s}+\frac{\partial Q^{i}}{\partial q^{s}} \mathrm{~d} q^{s}+\frac{\partial Q^{i}}{\partial t} \mathrm{~d} t\right)-\frac{\partial K}{\partial p_{l}} \mathrm{~d} p_{l} \wedge \mathrm{d} t-\frac{\partial K}{\partial q^{l}} \mathrm{~d} q^{l} \wedge \mathrm{d} t \\
& =\frac{\partial P_{i}}{\partial p_{j}} \frac{\partial Q^{i}}{\partial p_{s}} \mathrm{~d} p_{j} \wedge \mathrm{d} p_{s}+\frac{\partial P_{i}}{\partial q^{j}} \frac{\partial Q^{i}}{\partial q^{s}} \mathrm{~d} q^{j} \wedge \mathrm{d} q^{s}+\left(\frac{\partial P_{i}}{\partial p_{j}} \frac{\partial Q^{i}}{\partial q^{s}}-\frac{\partial P_{i}}{\partial q^{s}} \frac{\partial Q^{i}}{\partial p_{j}}\right) \mathrm{d} p_{j} \wedge \mathrm{d} q^{s} \\
& +\left(\frac{\partial P_{i}}{\partial p_{j}} \frac{\partial Q^{i}}{\partial t}-\frac{\partial P_{i}}{\partial t} \frac{\partial Q^{i}}{\partial p_{j}}-\frac{\partial K}{\partial p_{j}}\right) \mathrm{d} p_{j} \wedge \mathrm{d} t+\left(\frac{\partial P_{i}}{\partial q^{j}} \frac{\partial Q^{i}}{\partial t}-\frac{\partial P_{i}}{\partial t} \frac{\partial Q^{i}}{\partial q^{j}}-\frac{\partial K}{\partial q^{j}}\right) \mathrm{d} q^{j} \wedge \mathrm{d} t \\
& =-\frac{1}{2}\left[p_{j}, p_{s}\right] \mathrm{d} p_{j} \wedge \mathrm{d} p_{s}-\frac{1}{2}\left[q^{j}, q^{s}\right] \mathrm{d} q^{j} \wedge \mathrm{d} q^{s}-\left[p_{j}, q^{s}\right] \mathrm{d} p_{j} \wedge \mathrm{d} q^{s} \\
& +\left(-\left[p_{j}, t\right]-\frac{\partial K}{\partial p_{j}}\right) \mathrm{d} p_{j} \wedge \mathrm{d} t+\left(-\left[q^{j}, t\right]-\frac{\partial K}{\partial q^{j}}\right) \mathrm{d} q^{j} \wedge \mathrm{d} t,
\end{aligned}
$$

where $[u, v]$ denotes the Lagrange bracket, defined by $[u, v]=\frac{\partial Q^{i}}{\partial u} \frac{\partial P_{i}}{\partial v}-\frac{\partial P_{i}}{\partial u} \frac{\partial Q^{i}}{\partial v}$. Hence,

$$
\begin{aligned}
\mathbf{X}\lrcorner\left(\mathrm{d} P_{i} \wedge \mathrm{d} Q^{i}-d K \wedge d t\right) & =\left(\left[p_{j}, p_{l}\right] \frac{\partial H}{\partial q^{j}}-\left[q^{j}, p_{l}\right] \frac{\partial H}{\partial p_{j}}-\left[t, p_{l}\right]+\frac{\partial K}{\partial p_{l}}\right) \mathrm{d} p_{l} \\
& +\left(\left[q^{l}, q^{j}\right] \frac{\partial H}{\partial p_{j}}-\left[q^{l}, p_{j}\right] \frac{\partial H}{\partial q^{j}}-\left[t, q^{l}\right]+\frac{\partial K}{\partial q^{l}}\right) \mathrm{d} q^{l} \\
& +\left[\left(\frac{\partial K}{\partial p_{j}}-\left[t, p_{j}\right]\right) \frac{\partial H}{\partial q^{j}}+\left(\left[t, q^{j}\right]-\frac{\partial K}{\partial q^{j}}\right) \frac{\partial H}{\partial p_{j}}\right] \mathrm{d} t .
\end{aligned}
$$

Thus, $\mathbf{X}\lrcorner\left(\mathrm{d} P_{i} \wedge \mathrm{d} Q^{i}-\mathrm{d} K \wedge \mathrm{d} t\right)=0$ if and only if

$$
\begin{array}{r}
{\left[p_{j}, p_{l}\right] \frac{\partial H}{\partial q^{j}}-\left[q^{j}, p_{l}\right] \frac{\partial H}{\partial p_{j}}-\left[t, p_{l}\right]+\frac{\partial K}{\partial p_{l}}=0,} \\
{\left[q^{l}, q^{j}\right] \frac{\partial H}{\partial p_{j}}-\left[q^{l}, p_{j}\right] \frac{\partial H}{\partial q^{j}}-\left[t, q^{l}\right]+\frac{\partial K}{\partial q^{l}}=0,} \\
\left(\frac{\partial K}{\partial p_{j}}-\left[t, p_{j}\right]\right) \frac{\partial H}{\partial q^{j}}+\left(\left[t, q^{j}\right]-\frac{\partial K}{\partial q^{j}}\right) \frac{\partial H}{\partial p_{j}}=0 .
\end{array}
$$

We can check that if the first and the second equations are satisfied then the third equation also holds. So, we can forget the third one and we rewrite the first two equations in the form

$$
\frac{\partial K}{\partial p_{l}}=\left[p_{l}, p_{j}\right] \frac{\partial H}{\partial q^{j}}-\left[p_{l}, q^{j}\right] \frac{\partial H}{\partial p_{j}}+\left[t, p_{l}\right] \quad \text { and } \quad \frac{\partial K}{\partial q^{l}}=\left[q^{l}, p_{j}\right] \frac{\partial H}{\partial q^{j}}-\left[q^{l}, q^{j}\right] \frac{\partial H}{\partial p_{j}}+\left[t, q^{l}\right] .
$$

The existence of a function $K$ satisfying Eqs. (1) is equivalent to the condition that the 1-form

$$
\left(\left[p_{l}, p_{j}\right] \frac{\partial H}{\partial q^{j}}-\left[p_{l}, q^{j}\right] \frac{\partial H}{\partial p_{j}}+\left[t, p_{l}\right]\right) \mathrm{d} p_{l}+\left(\left[q^{l}, p_{j}\right] \frac{\partial H}{\partial q^{j}}-\left[q^{l}, q^{j}\right] \frac{\partial H}{\partial p_{j}}+\left[t, q^{l}\right]\right) \mathrm{d} q^{l}
$$

be exact. Letting $\left(x^{1}, x^{2}, \ldots, x^{2 n}\right) \equiv\left(q^{1}, \ldots, q^{n}, p_{1}, \ldots, p_{n}\right)$, the Hamilton equations are given by $\dot{x}^{\mu}=\epsilon^{\mu \nu}\left(\partial H / \partial x^{\nu}\right)$, where the lower case Greek letters run from 1 to $2 n$ and $\left(\epsilon^{\mu \nu}\right)$ is the $2 n \times 2 n$ matrix $\left(\begin{array}{cc}0 & I \\ -I & 0\end{array}\right)\left(\epsilon^{\mu \nu}\right.$ is the entry in the $\mu$-th row and the $\nu$-th column). Then, using this notation, we rewrite Eqs. (1) as

$$
\frac{\partial K}{\partial x^{\alpha}}=\epsilon^{\mu \nu}\left[x^{\mu}, x^{\alpha}\right] \frac{\partial H}{\partial x^{\nu}}+\left[t, x^{\alpha}\right]
$$


and the exactness condition of the 1-form mentioned above is $\partial^{2} K / \partial x^{\beta} \partial x^{\alpha}=\partial^{2} K / \partial x^{\alpha} \partial x^{\beta}$ which amounts to

$$
\epsilon^{\mu \nu} \frac{\partial\left[x^{\beta}, x^{\alpha}\right]}{\partial x^{\mu}} \frac{\partial H}{\partial x^{\nu}}+\epsilon^{\mu \nu}\left[x^{\nu}, x^{\beta}\right] \frac{\partial^{2} H}{\partial x^{\alpha} \partial x^{\mu}}-\epsilon^{\mu \nu}\left[x^{\nu}, x^{\alpha}\right] \frac{\partial^{2} H}{\partial x^{\beta} \partial x^{\mu}}+\frac{\partial\left[x^{\beta}, x^{\alpha}\right]}{\partial t}=0 .
$$

This equation is a necessary and sufficient condition for the local existence of a function $K$, such that the coordinate transformation $\left(q^{i}, p_{i}\right) \mapsto\left(Q^{i}, P_{i}\right)$ is a canonoid transformation with new Hamiltonian function $K$. The coordinate transformation is canonical if and only if the Lagrange brackets $\left[x^{\mu}, x^{\nu}\right]$ are given by $\left[x^{\mu}, x^{\nu}\right]=\epsilon^{\mu \nu}$.

\section{Bi-Hamiltonian systems}

In this section we begin by reviewing the standard treatment of bi-Hamiltonian systems. Let $(M, \omega, H)$ be an autonomous Hamiltonian system. If $\gamma$ is a second symplectic structure over $M$, we can define a $(1,1)$-tensor field $S$ on $M$ by the relation $\gamma(\mathbf{Y}, \mathbf{Z})=\omega(S \mathbf{Y}, \mathbf{Z})$ for any pair of vector fields $\mathbf{Y}, \mathbf{Z}$. The following definition is due to F. Magri and C. Morosi [4]: We say that $\gamma$ is compatible with the given Hamiltonian system if $£_{\mathbf{X}} \gamma=0$ and $N_{S}=0$, where $\mathbf{X}$ is the vector field that defines the dynamics of the system (that is, $\mathbf{X}\lrcorner \omega=-\mathrm{d} H$ ), $£_{\mathbf{X}}$ denotes the Lie derivative along $\mathbf{X}$, and $N_{S}$ is the Nijenhuis tensor of $S$, defined by

$$
N_{S}(\mathbf{Y}, \mathbf{Z})=[S \mathbf{Y}, S \mathbf{Z}]-S[S \mathbf{Y}, \mathbf{Z}]-S[\mathbf{Y}, S \mathbf{Z}]+S^{2}[\mathbf{Y}, \mathbf{Z}]
$$

Since the Lie derivatives of $\omega$ and $\gamma$ with respect to $\mathbf{X}$ are equal to zero, the Lie derivative of $S$ with respect to $\mathbf{X}$ is also equal to zero, which, in terms of an arbitrary coordinate system $x^{\alpha}$ of $M$ reads, $X^{\mu} \partial_{\mu} S_{\beta}^{\alpha}+S_{\mu}^{\alpha} \partial_{\beta} X^{\mu}-S_{\beta}^{\mu} \partial_{\mu} X^{\alpha}=0$, where $\partial_{\mu} \equiv \partial / \partial x^{\mu}$. Introducing the matrix $U=\left(U_{\beta}^{\alpha}\right)$, with $U_{\beta}^{\alpha} \equiv \partial_{\beta} X^{\alpha}\left(U_{\beta}^{\alpha}\right.$ is the entry in the $\alpha$-th row and the $\beta$-th column of the matrix $U$ ), the preceding equation can be expressed as the matrix equation $\mathrm{d} S / \mathrm{d} t=U S-S U=[U, S]$, where $S=\left(S_{\beta}^{\alpha}\right)$ is the matrix formed with the components of $S$. This last equation implies that the traces of the powers of the matrix $S$ yield $n=(1 / 2) \operatorname{dim} M$ constants of motion in involution that do not depend explicitly on time [6,7,14] (see Eq. (4) below).

We are interested in extending these results to non-autonomous Hamiltonian systems. To this end, we consider a canonoid transformation $Q^{i}=Q^{i}\left(x^{\mu}, t\right), P_{i}=P_{i}\left(x^{\mu}, t\right)$ as in Sec. 2, and we define the tensor field $S=S_{\beta}^{\alpha}\left(\partial / \partial x^{\alpha}\right) \otimes \mathrm{d} x^{\beta}$ given in the canonical coordinates $x^{\mu}$ by $S_{\beta}^{\alpha}=\epsilon^{\alpha \lambda} T_{\beta \lambda}$, where $T_{\mu \nu} \equiv\left[x^{\mu}, x^{\nu}\right]$. Then we can see that the functions $K_{m}=(1 / m) \operatorname{tr}\left(S^{m}\right)$, $m \geqslant 1$, are constants of motion and, therefore, the eigenvalues of $S$ are also constants of motion. For this, we define $U_{\beta}^{\alpha}=$ $\epsilon^{\alpha \lambda}\left(\partial^{2} H / \partial x^{\lambda} \partial x^{\beta}\right)$ and we note that Eq. (2) is equivalent to $\mathrm{d} S_{\beta}^{\lambda} / \mathrm{d} t+S_{\nu}^{\lambda} U_{\beta}^{\nu}-U_{\mu}^{\lambda} S_{\beta}^{\mu}=0$, indeed,

$$
\begin{aligned}
\frac{\mathrm{d} S_{\beta}^{\lambda}}{\mathrm{d} t} & +S_{\nu}^{\lambda} U_{\beta}^{\nu}-U_{\mu}^{\lambda} S_{\beta}^{\mu} \\
& =\epsilon^{\lambda \alpha} \frac{\partial\left[x^{\beta}, x^{\alpha}\right]}{\partial t}+\epsilon^{\lambda \alpha} \frac{\partial\left[x^{\beta}, x^{\alpha}\right]}{\partial x^{\mu}} \frac{\mathrm{d} x^{\mu}}{\mathrm{d} t}+\epsilon^{\lambda \alpha}\left[x^{\nu}, x^{\alpha}\right] \epsilon^{\nu \mu} \frac{\partial^{2} H}{\partial x^{\mu} \partial x^{\beta}}-\epsilon^{\lambda \alpha} \frac{\partial^{2} H}{\partial x^{\alpha} \partial x^{\mu} \epsilon^{\mu \nu}\left[x^{\beta}, x^{\nu}\right]} \\
& =\epsilon^{\lambda \alpha}\left(\frac{\partial\left[x^{\beta}, x^{\alpha}\right]}{\partial t}+\frac{\partial\left[x^{\beta}, x^{\alpha}\right]}{\partial x^{\mu}} \epsilon^{\mu \nu} \frac{\partial H}{\partial x^{\nu}}+\epsilon^{\nu \mu}\left[x^{\nu}, x^{\alpha}\right] \frac{\partial^{2} H}{\partial x^{\mu} \partial x^{\beta}}-\epsilon^{\mu \nu}\left[x^{\beta}, x^{\nu}\right] \frac{\partial^{2} H}{\partial x^{\alpha} \partial x^{\mu}}\right) \\
& =\epsilon^{\lambda \alpha}\left(\frac{\partial\left[x^{\beta}, x^{\alpha}\right]}{\partial t}+\epsilon^{\mu \nu} \frac{\partial\left[x^{\beta}, x^{\alpha}\right]}{\partial x^{\mu}} \frac{\partial H}{\partial x^{\nu}}-\epsilon^{\mu \nu}\left[x^{\nu}, x^{\alpha}\right] \frac{\partial^{2} H}{\partial x^{\mu} \partial x^{\beta}}+\epsilon^{\mu \nu}\left[x^{\nu}, x^{\beta}\right] \frac{\partial^{2} H}{\partial x^{\alpha} \partial x^{\mu}}\right)
\end{aligned}
$$

or, in matrix form, $\mathrm{d} S / \mathrm{d} t=U S-S U=[U, S]$. Then, making use of the cyclic property of the trace,

$$
\begin{aligned}
\frac{\mathrm{d}}{\mathrm{d} t} K_{m} & =\frac{1}{m} \operatorname{tr}\left(\frac{\mathrm{d}}{\mathrm{d} t} S^{m}\right)=\frac{1}{m} \operatorname{tr}\left(\frac{\mathrm{d} S}{\mathrm{~d} t} S^{m-1}+S \frac{\mathrm{d} S}{\mathrm{~d} t} S^{m-2}+\cdots+S^{m-1} \frac{\mathrm{d} S}{\mathrm{~d} t}\right) \\
& =\operatorname{tr}\left(\frac{\mathrm{d} S}{\mathrm{~d} t} S^{m-1}\right)=\operatorname{tr}\left([U, S] S^{m-1}\right)=0 .
\end{aligned}
$$

It may be remarked that the traces $K_{m}$ may be trivial constants (even all of them) and that they may not be in involution. The maximum number of functionally independent constants of motion obtained in this way is $n$. This result follows from the fact that the characteristic polynomial of the product of two antisymmetric $2 n \times 2 n$ matrices is the square of a polynomial of degree $n$ (see Ref. [15]).

In order to have an integrable system, it would be desirable to have $n$ functionally independent constants of motion in involution. As we shall show below, following the discussion presented in Refs. [6, 7, 14] for the case of autonomous systems, we show that the vanishing of the Nijenhuis tensor of $S$ (which now may depend explicitly on time) implies that the constants of motion $K_{m}$ are in involution. First, we note that the conditions

$$
N_{\alpha \beta}^{\lambda}\left(S^{l-1}\right)_{\lambda}^{\beta}=0,
$$


where $N_{\alpha \beta}^{\lambda}$ are the components of the Nijenhuis tensor of $S,\left(S^{m}\right)_{\lambda}^{\beta}$ are the entries of the matrix $S^{m}$, and $l$ is an integer greater than zero, are equivalent to

$$
S_{\alpha}^{\nu} \partial_{\nu} K_{l}=\partial_{\alpha} K_{l+1} .
$$

Equations (6) are called the Lenard recursion relations (see, e.g., Ref. [14]). In fact, we have the following chain of equivalent equations, for $l \geqslant 1$,

$$
\begin{array}{r}
S_{\alpha}^{\nu} \partial_{\nu} K_{l}-\partial_{\alpha} K_{l+1}=0 \\
\Leftrightarrow \frac{1}{l} S_{\alpha}^{\nu} \partial_{\nu} \operatorname{tr}\left(S^{l}\right)-\frac{1}{l+1} \partial_{\alpha} \operatorname{tr}\left(S^{l+1}\right)=0 \\
\Leftrightarrow S_{\alpha}^{\nu}\left(\partial_{\nu} S_{\beta}^{\lambda}\right)\left(S^{l-1}\right)_{\lambda}^{\beta}-\left(\partial_{\alpha} S_{\beta}^{\nu}\right)\left(S^{l}\right)_{\nu}^{\beta}=0 \\
\Leftrightarrow S_{\alpha}^{\nu}\left(\partial_{\nu} S_{\beta}^{\lambda}\right)\left(S^{l-1}\right)_{\lambda}^{\beta}-S_{\nu}^{\lambda}\left(\partial_{\alpha} S_{\beta}^{\nu}\right)\left(S^{l-1}\right)_{\lambda}^{\beta}=0 \\
\Leftrightarrow S_{\alpha}^{\nu}\left(\partial_{\nu} S_{\beta}^{\lambda}\right)\left(S^{l-1}\right)_{\lambda}^{\beta}-S_{\nu}^{\lambda}\left(\partial_{\alpha} S_{\beta}^{\nu}\right)\left(S^{l-1}\right)_{\lambda}^{\beta}+\left(S^{l}\right)_{\nu}^{\beta} \partial_{\beta} S_{\alpha}^{\nu}-\left(S^{l}\right)_{\lambda}^{\nu} \partial_{\nu} S_{\alpha}^{\lambda}=0 \\
\Leftrightarrow S_{\alpha}^{\nu}\left(\partial_{\nu} S_{\beta}^{\lambda}\right)\left(S^{l-1}\right)_{\lambda}^{\beta}-S_{\nu}^{\lambda}\left(\partial_{\alpha} S_{\beta}^{\nu}\right)\left(S^{l-1}\right)_{\lambda}^{\beta}+S_{\nu}^{\lambda}\left(\partial_{\beta} S_{\alpha}^{\nu}\right)\left(S^{l-1}\right)_{\lambda}^{\beta}-S_{\beta}^{\nu}\left(\partial_{\nu} S_{\alpha}^{\lambda}\right)\left(S^{l-1}\right)_{\lambda}^{\beta}=0 \\
\Leftrightarrow\left[S_{\alpha}^{\nu}\left(\partial_{\nu} S_{\beta}^{\lambda}\right)-S_{\nu}^{\lambda}\left(\partial_{\alpha} S_{\beta}^{\nu}\right)+S_{\nu}^{\lambda}\left(\partial_{\beta} S_{\alpha}^{\nu}\right)-S_{\beta}^{\nu}\left(\partial_{\nu} S_{\alpha}^{\lambda}\right)\right]\left(S^{l-1}\right)_{\lambda}^{\beta}=0 .
\end{array}
$$

The conclusion now follows, using the fact that $S_{\alpha}^{\nu}\left(\partial_{\nu} S_{\beta}^{\lambda}\right)-S_{\nu}^{\lambda}\left(\partial_{\alpha} S_{\beta}^{\nu}\right)+S_{\nu}^{\lambda}\left(\partial_{\beta} S_{\alpha}^{\nu}\right)-S_{\beta}^{\nu}\left(\partial_{\nu} S_{\alpha}^{\lambda}\right)$ is the component $N_{\alpha \beta}^{\lambda}$ of the Nijenhuis tensor of $S$.

As in the autonomous case, the Lenard recursion relations imply that the functions $K_{m}$ are in involution. The proof is identical to that of the autonomous case. Noting that the Lenard recursion relations are equivalent to

$$
\epsilon^{\mu \nu} \partial_{\nu} K_{l}=T^{\mu \nu} \partial_{\nu} K_{l+1},
$$

where the functions $T^{\mu \nu}$ are defined by $T^{\mu \nu} T_{\mu \alpha}=\delta_{\alpha}^{\nu}$ (the matrix $\left(T^{\mu \nu}\right)$ is minus the inverse of $\left(T_{\mu \nu}\right)$ and, therefore, is also antisymmetric), we have

$$
\left\{K_{s}, K_{l}\right\}=\varepsilon^{\mu \nu}\left(\partial_{\mu} K_{s}\right)\left(\partial_{\nu} K_{l}\right)=T^{\mu \nu}\left(\partial_{\mu} K_{s+1}\right)\left(\partial_{\nu} K_{l}\right)=\left(\partial_{\mu} K_{s+1}\right) \varepsilon^{\mu \nu}\left(\partial_{\nu} K_{l-1}\right)=\left\{K_{s+1}, K_{l-1}\right\} .
$$

Hence, if $i>j$,

$$
\left\{K_{i}, K_{j}\right\}=\left\{K_{i-1}, K_{j+1}\right\}=\cdots=\left\{K_{i-(i-j-1)}, K_{j+(i-j-1)}\right\}=\left\{K_{j}, K_{i}\right\},
$$

which means that $\left\{K_{i}, K_{j}\right\}=0$, for all $i, j$. Thus, when $S$ has exactly $n$ different eigenvalues and $N_{S}=0$, we have $n$ constants of motion in involution that may depend explicitly on time (see the examples below).

So far we have only considered the Nijenhuis torsion tensor, but there is another torsion tensor, the Haantjes tensor, which is also relevant [16]. A new formulation of classical integrability based on Haantjes operators, namely $(1,1)$-tensor fields with vanishing Haantjes torsion tensor has been presented in Ref. [17]. If $N_{L}$ is the Nijenhuis torsion tensor of a $(1,1)$-tensor field $L$ then the Haantjes torsion tensor of $L$ is defined by

$$
\mathcal{H}_{L}(\mathbf{Y}, \mathbf{Z})=L^{2} N_{L}(\mathbf{Y}, \mathbf{Z})+N_{L}(L \mathbf{Y}, L \mathbf{Z})-L\left(N_{L}(\mathbf{Y}, L \mathbf{Z})+N_{L}(L \mathbf{Y}, \mathbf{Z})\right)
$$

for any pair of vector fields $\mathbf{Y}, \mathbf{Z}$. It is obvious that if the Nijenhuis torsion tensor of $L$ vanishes then the Haantjes torsion tensor of $L$ also vanishes, but the converse is not true in general [16]. The components of the Haantjes torsion tensor of $L$ in local coordinates are

$$
\left(\mathcal{H}_{L}\right)_{\alpha \beta}^{\lambda}=L_{\nu}^{\lambda} L_{\mu}^{\nu}\left(N_{L}\right)_{\alpha \beta}^{\mu}+\left(N_{L}\right)_{\nu \mu}^{\lambda} L_{\alpha}^{\nu} L_{\beta}^{\mu}-L_{\nu}^{\lambda}\left(\left(N_{L}\right)_{\mu \beta}^{\nu} L_{\alpha}^{\mu}+\left(N_{L}\right)_{\alpha \mu}^{\nu} L_{\beta}^{\mu}\right) .
$$

In example 2, below, we show that even if the Nijenhuis torsion tensor and the Haantjes torsion tensor are different from zero, it is possible to have that the constants of motion found by means of a canonoid transformation can be in involution.

In the autonomous case, $S$ can be viewed as a linear transformation from the tangent space to $M$ at a point $p$ into itself (as in Eq. (3)), and it has been established that the eigenvectors of $S$ for a given eigenvalue define an involutive (and, hence, locally totally integrable) distribution (see Ref. [3]). This result can be extended to the non-autonomous case: If the vector fields $\mathbf{Y}=Y^{\mu} \partial_{\mu}$ and $\mathbf{Z}=Z^{\mu} \partial_{\mu}$ are eigenvector fields of $S$ with eigenvalue $\lambda$ then, assuming that the Nijenhuis tensor of $S$ is equal to zero, from (3) we have

$$
\mathbf{0}=[\lambda \mathbf{Y}, \lambda \mathbf{Z}]-S[\lambda \mathbf{Y}, \mathbf{Z}]-S[\mathbf{Y}, \lambda \mathbf{Z}]+S^{2}[\mathbf{Y}, \mathbf{Z}]=\lambda^{2}[\mathbf{Y}, \mathbf{Z}]-2 \lambda S[\mathbf{Y}, \mathbf{Z}]+S^{2}[\mathbf{Y}, \mathbf{Z}]=(S-\lambda)^{2}[\mathbf{Y}, \mathbf{Z}],
$$


which implies that $(S-\lambda)[\mathbf{Y}, \mathbf{Z}]=0$ (since $S$ is diagonalizable), that is, $[\mathbf{Y}, \mathbf{Z}]$ is an eigenvector field of $S$ with eigenvalue $\lambda$ then the eigenvectors of $S$ for a given eigenvalue define an involutive distribution.

We finish this section by remarking that the equation $\mathrm{d} S / \mathrm{d} t=[U, S]$ is fundamental for this work, some authors call it a Lax equation (see for example Refs. [1,6]). Another concept related to this equation is a Lax pair; for a Hamiltonian system with $n$ degrees of freedom, a Lax pair is a pair of matrices $L, M$ of order $n \times n$, functions of the phase space of the system, such that Hamilton equations can be written as $\mathrm{d} L / \mathrm{d} t=[M, L]$ (see Ref. [1]). If we have a Lax pair $L, M$ for a Hamiltonian system then the eigenvalues of $L$ are constants of motion; note that the pair of matrices $S, U$ in this work is not a Lax pair, since the Hamilton equations are not necessarily given by $\mathrm{d} S / \mathrm{d} t=[U, S]$, nevertheless, as was shown, the eigenvalues of $S$ are constants of motion.

\section{Examples}

In this section we present three examples, the first one shows a canonoid transformation where the Nijenhuis tensor vanishes. The second and the third ones give canonoid transformations which lead to constants of motion that are in involution even though the Nijenhuis tensor does not vanish.

\subsection{Example 1}

Consider a Hamiltonian system with canonical coordinates $\left(q^{1}, q^{2}, p_{1}, p_{2}\right)$ and Hamiltonian function

$$
H\left(q^{1}, q^{2}, p_{1}, p_{2}, t\right)=-5 t q^{1}+3 t^{2} q^{2}+p_{2} \sin t .
$$

One can check that the coordinate transformation

$$
\begin{aligned}
& Q^{1}=\frac{1}{2}\left(q^{1}\right)^{2}, \\
& Q^{2}=p_{2}+t^{3}+\mathrm{e}^{t}, \\
& P_{1}=p_{1}, \\
& P_{2}=p_{2}\left(1-q^{2}-\cos t\right)-t^{3} q^{2}-t^{3} \cos t-\mathrm{e}^{t},
\end{aligned}
$$

is canonoid with Hamiltonian function

$$
K=-5 t Q^{1}+\left(3 t^{2}+\mathrm{e}^{t}\right) Q^{2}+\mathrm{e}^{t} P_{2}
$$

(That is, $\dot{Q}^{i}=\partial K / \partial P_{i}$ and $\dot{P}_{i}=-\partial K / \partial Q^{i}$ ).

The matrix $S=\left(S_{\beta}^{\alpha}\right)$ is given by

$$
S=\left(\begin{array}{cccc}
{\left[q^{1}, p_{1}\right]} & {\left[q^{2}, p_{1}\right]} & 0 & {\left[p_{2}, p_{1}\right]} \\
{\left[q^{1}, p_{2}\right]} & {\left[q^{2}, p_{2}\right]} & {\left[p_{1}, p_{2}\right]} & 0 \\
0 & {\left[q^{1}, q^{2}\right]} & {\left[q^{1}, p_{1}\right]} & {\left[q^{1}, p_{2}\right]} \\
{\left[q^{2}, q^{1}\right]} & 0 & {\left[q^{2}, p_{1}\right]} & {\left[q^{2}, p_{2}\right]}
\end{array}\right)=\left(\begin{array}{cccc}
q^{1} & 0 & 0 & 0 \\
0 & p_{2}+t^{3} & 0 & 0 \\
0 & 0 & q^{1} & 0 \\
0 & 0 & 0 & p_{2}+t^{3}
\end{array}\right)
$$

and the (multiplicity two) eigenvalues of $S$ are

$$
\lambda_{1}=q^{1} \quad \text { and } \quad \lambda_{2}=p_{2}+t^{3} .
$$

One can verify directly that $\lambda_{1}$ and $\lambda_{2}$ are constants of motion in involution, which also follows from the fact that the Nijenhuis tensor of $S$ vanishes. Furthermore, they are functionally independent.

It may be remarked that, in all cases, det $S$ must be different from zero everywhere. In this example det $S$ is zero at the points $q^{1}=0$, and $p_{2}+t^{3}=0$, but this is a consequence of the fact that at these points the coordinate transformation considered here is singular.

\subsection{Example 2}

Consider a Hamiltonian system with canonical coordinates $\left(q^{1}, q^{2}, p_{1}, p_{2}\right)$ and Hamiltonian function

$$
H\left(q^{1}, q^{2}, p_{1}, p_{2}, t\right)=\left(t^{3}-5 t\right) p_{1}+3 t^{2} p_{2}-t q^{1}-q^{2} \sin t .
$$


One can check that the coordinate transformation

$$
\begin{aligned}
Q^{1} & =q^{2} \\
Q^{2} & =p_{2}-2 p_{1}+t^{2} \\
P_{1} & =p_{1}\left(\frac{5}{2} t^{2}-\frac{1}{4} t^{4}\right)+q^{1}\left(p_{1}-\frac{1}{2} t^{2}\right)+\left(p_{2}+\cos t\right)^{2}-q^{2}+\frac{1}{8} t^{6}-\frac{5}{4} t^{4}+t^{3}+\sin t^{2}, \\
P_{2} & =-p_{2}-\cos t-4 \mathrm{e}^{t},
\end{aligned}
$$

is a canonoid transformation with Hamiltonian function

$$
K\left(Q^{1}, Q^{2}, P_{1}, P_{2}, t\right)=3 t^{2} P_{1}+P_{2} \sin t-2 t Q^{1} \cos t^{2}+4 \mathrm{e}^{t} Q^{2} .
$$

Then

$$
S=\left(\begin{array}{cccc}
0 & \frac{5}{2} t^{2}-\frac{1}{4} t^{4}+q^{1} & 0 & -2 \\
0 & 2\left(p_{2}+\cos t\right) & 2 & 0 \\
0 & -p_{1}+\frac{1}{2} t^{2} & 0 & 0 \\
p_{1}-\frac{1}{2} t^{2} & 0 & \frac{5}{2} t^{2}-\frac{1}{4} t^{4}+q^{1} & 2\left(p_{2}+\cos t\right)
\end{array}\right)
$$

and the eigenvalues of $S$ are

$$
\lambda_{1}=p_{2}+\cos t+\sqrt{\left(p_{2}+\cos t\right)^{2}+t^{2}-2 p_{1}}
$$

and

$$
\lambda_{2}=p_{2}+\cos t-\sqrt{\left(p_{2}+\cos t\right)^{2}+t^{2}-2 p_{1}},
$$

so that $K_{1}=4\left(p_{2}+\cos t\right)$ and $K_{2}=4 t^{2}-8 p_{1}+8\left(p_{2}+\cos t\right)^{2}$. One can verify that these are functionally independent constants of motion in involution. However, neither the Nijenhuis tensor nor the Haantjes tensor of $S$ vanish. For instance,

$$
N_{12}^{2}=S_{1}^{\lambda} \partial_{\lambda} S_{2}^{2}-S_{2}^{\lambda} \partial_{\lambda} S_{1}^{2}-S_{\lambda}^{2} \partial_{1} S_{2}^{\lambda}+S_{\lambda}^{2} \partial_{2} S_{1}^{\lambda}=S_{1}^{4} \partial_{4} S_{2}^{2}-0-0+0=2 p_{1}-t^{2}
$$

and

$$
\left(\mathcal{H}_{S}\right)_{13}^{1}=\left(2 t^{2}-4 p_{1}\right)\left(1+5 t^{2}-\frac{1}{2} t^{4}+2 q^{1}\right)
$$

The Lenard recursion relations also fail: We have $\partial_{1} K_{2}=0$, but

$$
S_{1}^{\lambda} \partial_{\lambda} K_{1}=S_{1}^{4} \partial_{4} K_{1}=4 p_{1}-2 t^{2} \neq 0
$$

\subsection{Example 3}

Consider the Hamiltonian system with canonical coordinates $\left(q^{1}, q^{2}, q^{3}, p_{1}, p_{2}, p_{3}\right)$ and Hamiltonian function

$$
H\left(q^{1}, q^{2}, q^{3}, p_{1}, p_{2}, p_{3}, t\right)=t p_{1}+\frac{1}{3} p_{2}{ }^{3}-\mathrm{e}^{t} p_{3}-5 q^{2}-q^{3} \sin t .
$$

The coordinate transformation

$$
\begin{aligned}
& Q^{1}=p_{2} \\
& Q^{2}=p_{1}^{2}+p_{3} \\
& Q^{3}=q^{3}+\mathrm{e}^{t}-t^{4} \\
& P_{1}=p_{1}\left(p_{2}-5 t\right)\left(q^{2}-\frac{1}{15} p_{2}{ }^{3}\right), \\
& P_{2}=q^{1}-p_{3}-t^{2} p_{1}-\mathrm{e}^{t}-\cos t \\
& P_{3}=q^{3}\left(p_{3}+\cos t+1\right)+\mathrm{e}^{t}\left(p_{3}+\cos t\right),
\end{aligned}
$$

is a canonoid transformation with Hamiltonian function

$$
K\left(Q^{1}, Q^{2}, Q^{3}, P_{1}, P_{2}, P_{3}, t\right)=5 P_{1}+P_{2} \sin t-4 t^{3} P_{3}+\mathrm{e}^{t} Q^{2}+\mathrm{e}^{t} Q^{3} .
$$


Then one finds that the matrix $\left(S_{\beta}^{\alpha}\right)$ is

$$
S=\left(\begin{array}{cccccc}
-2 p_{1} & 0 & 0 & 0 & \left(q^{2}-\frac{1}{15} p_{2}^{3}\right)\left(p_{2}-5 t\right) & 2 p_{1}-t^{2} \\
0 & p_{1}\left(5 t-p_{2}\right) & 0 & \left(q^{2}-\frac{1}{15} p_{2}{ }^{3}\right)\left(5 t-p_{2}\right) & 0 & 0 \\
-1 & 0 & q^{3}+\mathrm{e}^{t} & t^{2}-2 p_{1} & 0 & 0 \\
0 & 0 & 0 & -2 p_{1} & 0 & -1 \\
0 & 0 & 0 & 0 & p_{1}\left(5 t-p_{2}\right) & 0 \\
0 & 0 & 0 & 0 & 0 & q^{3}+\mathrm{e}^{t}
\end{array}\right) .
$$

The eigenvalues of $S$ are

$$
\lambda_{1}=-2 p_{1}, \quad \lambda_{2}=p_{1}\left(5 t-p_{2}\right), \quad \lambda_{3}=q^{3}+\mathrm{e}^{t},
$$

and one can verify that they are functionally independent constants of motion in involution.

On the other hand, as in the previous example, the Nijenhuis tensor does not vanish; for instance, we have

$$
N_{45}^{5}=2 p_{1}\left(p_{2}-5 t\right)-p_{1}\left(p_{2}-5 t\right)^{2} .
$$

\section{Conclusions}

We have shown that it is possible to extend the existing results about bi-Hamiltonian systems to the non-autonomous case, that is, for a given Hamiltonian system with Hamiltonian function possibly depending explicitly on time, and with Hamilton equations expressed in terms of a set of canonical coordinates, or of a Poisson bracket. By starting from a canonoid transformation that may depend explicitly on time, we get a second Poisson bracket related to the first one by means of a tensor field $S_{\beta}^{\alpha}$ and we find constants of motion of the given system by taking the trace of the each power of the matrix $\left(S_{\beta}^{\alpha}\right)$. In addition, we showed that the vanishing of the Nijenhuis torsion tensor of the tensor field $S_{\beta}^{\alpha}$ is a sufficient but not necessary condition for such constants of motion to be in involution. Finally we illustrated these results with some examples.

\section{Acknowledgements}

The authors wish to acknowledge the referee for useful comments and for pointing out Refs. [16,17] to them. One of the authors (R. Azuaje) wishes to thank CONACYT (México) for financial support through a fellowship.

1. O. Babelon, D. Bernard and M. Talon, Introduction to Classical Integrable Systems (Cambridge Monographs on Mathematical Physics). (Cambridge University Press, New York, 2003). https://doi.org/10.1017/CB09780511535024

2. V. Arnold, V. Kozlov and A. Neishtadt, Mathematical Aspects of Classical and Celestial Mechanics, 3rd ed. (Springer-Verlag, Berlin, Heidelberg, 2006). https://doi.org/10.1007/ 978-3-540-48926-9

3. V.S. Gerdjikov, G. Vilasi and A.B. Yanovski. Integrable Hamiltonian Hierarchies, Lect. Notes Phys. 748. (Springer, Berlin, 2008). https://doi.org/10.1007/ 978-3-540-77054-1

4. F. Magri and C. Morosi, Quaderno S 19, Università degli Studi di Milano (1984).

5. F. Magri, J. Math. Phys. 19 (1978) 1156-1162. https:// doi.org/10.1063/1.523777

6. A. Das, Integrable Models. (World Scientific, Singapore, 1989). https://doi.org/10.1142/0858

7. R. Brouzet, J. Math. Phys. 34 (1993) 1309. https://doi. org/10.1063/1.530212

8. G. Rastelli and M. Santoprete, Journal of Geometric Mechanics
7 (2015) 4. https://doi.org/10.3934/jgm.2015. 7.483

9. L.J. Negri, L.C. Oliveira, and J.M. Teixeira, J. Math. Phys. 28 (1987) 2369.https://doi.org/10.1063/1.527772

10. J.F. Cariñena, F. Falceto and M.F. Rañada, Journal of Geometric Mechanics 5 (2013) 151. https://doi.org/10. 3934 / jgm.2013.5.151

11. J.F. Cariñena and M.F. Rañada, J. Math. Phys. 30 (1989) 2258. https://doi.org/10.1063/1.528552

12. G.F. Torres del Castillo, Differentiable Manifolds: A Theoretical Physics Approach, 2nd ed. (Birkhäuser, Cham, 2020). https://doi.org/10.1007/978-3-030-45193-6

13. G.F. Torres del Castillo, An Introduction to Hamiltonian Mechanics (Birkhäuser, Cham, 2018). https://doi.org/ 10.1007/978-3-319-95225-3

14. Y. Kosmann-Schwarzbach and F. Magri, J. Math. Phys. 37 (1996) 6173. https://doi.org/10.1063/1.531771

15. H. Eves, Elementary Matrix Theory (Dover, New York, 1980). Sect. 3.6A.

16. Y. Kosmann-Schwarzbach, in: P. Kielanowski, A. Odzijewicz and E. Previato (eds) Geometric Methods in Physics XXXVI 
G.F. TORRES DEL CASTILLO AND R. AZUAJE

(Birkhäuser, Cham, 2019).https://doi.org/10.1007/

978-3-030-01156-7_18

atica (2021). https://doi.org/10.1007/

17. P. Tempesta and G. Tondo, Annali di Matems10231-021-01107-4 